\title{
Comparing age and sex trends of chlamydia, gonorrhoea, hepatitis and syphilis infections in Samoa in 2012 and 2017
}

\author{
Robert Carney, ${ }^{a}$ Michaela Howells, ${ }^{b}$ Aaone Tanumafili, a Athena Matalavea, ${ }^{a}$ Judith Gafa, ${ }^{a}$ Leausa Toleafoa Dr Take \\ Naseri $^{a}$ \\ Correspondence to Robert Carney (email: robertc@health.gov.ws)
}

\begin{abstract}
In Samoa, the seroprevalence rates of sexually transmitted infections other than HIV have been endemically high over the past decade, despite years of prevention programming. Odds ratio and $\chi^{2}$ tests were conducted to compare the rates of positivity of chlamydia, gonorrhoea, hepatitis B and C, and syphilis across age groups from 2012 and 2017 surveillance data in Samoa. Young people aged 15-19 years were significantly more likely to have a chlamydia infection compared to all other age groups in both 2012 and 2017. Hepatitis B infections were more common in males and those aged 30 and above in both 2012 and 2017. Hepatitis C had no significant differences in age, but it was more common in males in 2012 and more common in females in 2017. Older age groups (aged 45 and above) were more likely to have a positive syphilis test in both 2014 and 2017 when compared to those aged 15-24 years. The results of this analysis confirm previously observed trends in Samoa for younger age groups' prevalence of chlamydia and gonorrhoea, and for older age groups' prevalence of hepatitis B and C. But the analysis also unexpectedly found that older age groups (aged 45 and above) are more likely to test positive for syphilis (for years 2014 and 2017). Further studies are needed to assess behavioural risk factors associated with older populations in order to explain the increase in risk and to design interventions suited to this demographic.
\end{abstract}

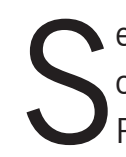
exually transmitted infections (STIs) are an ongoing health challenge in Samoa and across the Pacific. Samoa consists of four inhabited islands with a relatively small population of approximately 196000 people. $^{1}$ The country's close-knit community dynamic poses unique social barriers to voluntary testing and treatment, and population coverage remains low. ${ }^{2}$ Samoa has historically reported high rates of chlamydia and gonorrhoea and low rates of HIV and syphilis from cross-sectional antenatal prevalence studies. The first seroprevalence survey in the country, conducted in 2000 , found that chlamydia and trichomoniasis infections were common among pregnant women $(30.9 \%$ and $20.8 \%$, respectively), with a low prevalence of gonorrhoea and syphilis (3.3\% and $0.5 \%$, respectively). ${ }^{2}$ One analysis found that young women aged $<25$ years were three times more likely to have an STI than older women. ${ }^{3}$ Chlamydia is endemic among women, with those $<25$ years having greater positivity than those $\geq 25$ years $(26.1 \%$ compared to $11.9 \%){ }^{3}$ Women who are unmarried or are 18-24 years of age were more likely to test positive for chlamydia. ${ }^{4}$ Chlamydia infections are also associated with subfertility in Samoan women. ${ }^{5}$ Maternal and congenital syphilis in Samoa has increased since 2018. ${ }^{6}$ Untreated maternal syphilis has been associated with fetal loss, neonatal death, premature birth and lower birthweight. ${ }^{7}$ These results indicate significant health challenges for women of childbearing age.

The 2017 national positivity rates for chlamydia, gonorrhoea and syphilis $(24.2 \%, 5.6 \%$ and $1.04 \%$, respectively) are similar to previous prevalence rates. ${ }^{8}$ This suggests a persistently high prevalence of STIs, particularly chlamydia, for the past decade. Conversely, Samoa has previously reported the lowest rate of syphilis worldwide $(0 \%){ }^{8}$ However, syphilis has increased globally among heterosexuals and men who have sex with men. ${ }^{8}$ In Samoa, syphilis cases have steadily increased from 26 reported cases in 2015 to 78 cases in $2017 .{ }^{6}$

Although prevalence estimates were well established in previous studies, more recent data on sex and age are needed to inform intervention design. Using national

\footnotetext{
Samoa Ministry of Health, Apia, Samoa.

Department of Anthropology, University of North Carolina, Wilmington, North Carolina, United States of America. Submitted: 28 June 2019; Published: 31 March 2020

doi: 10.5365/wpsar.2019.10.2.004
} 
surveillance data, this analysis seeks to update the status of the epidemic by comparing positivity rates among sex and age groups from years 2012 and 2017.

\section{METHODS}

For a cross-sectional study, the data of all patients in the country who had an STI investigation (routine or suspect) from any provider were analysed for years 2012 ( $n=$ 18 804) and 2017 ( $n=48$ 898) to compare trends in the positive diagnosis of chlamydia, gonorrhoea, hepatitis $B$ and $C$, and syphilis by age and sex. Due to the low sample size in 2012, syphilis data were compared for 2014 ( $n=3865$ ) and 2017 ( $n=11$ 418). Patients with lost or insufficient specimens were excluded from the sample.

The HIV and STI Surveillance Database, which was used to construct the study sample, is managed by the Samoa Ministry of Health in the capital of Apia. The most complete full-year data sets (2012, 2014 and 2017) were used. Data are comprised of all diagnostic testing (both routine testing and suspect cases in the general population) that was recorded by the National Reference Laboratory (NRL) of Tupua Tamasese Meaole (TTM) Hospital, Apia. In 2017, all national diagnostic technologies were centrally housed in the NRL. All specimens collected in the country are processed by the NRL and recorded in the database. The assays used in the national testing algorithm include polymerase chain reaction (PCR) for chlamydia and gonorrhoea, rapid plasma reagin (RPR) and treponema pallidum haemagglutination (TPHA) tests for syphilis, hepatitis B surface antigen (HBsAg) tests for hepatitis B, the hepatitis $C$ antibody (anti-HCV) test for hepatitis $\mathrm{C}$. Clinicians collect and send specimens to $\mathrm{NRL}$ or refer the patient directly to the NRL for collection. Once specimens are processed, the requesting clinician and relevant authorities are notified, and the reporting is collated into the database.

We used Epi Info software (version 7.2.2.6) to estimate odds ratios and chi-square test $\left(\chi^{2)}\right.$ to analyse trends (extended Mantel-Haenzsel). Odds ratios are suitable estimations of the prevalence of a disease when infections are less than $10 \%{ }^{9,10}$ Samoa's syphilis positivity rates ranged from $0.13 \%$ in 2012 to $1.04 \%$ as of July 2018. ${ }^{11}$ Cases were compared based on sex (comparing males to females), age (comparing all other age groups to those aged 15-19 years) and the type of STI. Since there were only 10 cases in 2012, data for syphilis were taken from 2014 when there were sufficient cases $(n=18)$ by age to run the analysis without violating the assumption of the extended Mantel-Haenzsel tests ( $80 \%$ of cells have more than five cases). Additionally, syphilis age groups were adjusted from five-year to 10-year age groups ( $<45$ and $\geq 45$ ) for sample size, while other STI age groups are compared to those $<30$ years and $\geq 30+$ years.

\section{Sample}

The majority of tests come from routine testing $(76.0 \%$ in 2012 and 78.7\% in 2017, Annex). This includes testing of women attending antenatal care services, immigration screening for Samoan nationals and blood donation screening. Females represent more total tests than males (71.5\% compared to $28.5 \%$ in 2012 , and $56.7 \%$ versus $43.3 \%$ in 2017, respectively). There were 7861 tests in 2012 and 11152 in 2017 in individuals < 30. Testing increased for individuals age $\geq 30$ years (6428 tests in 2012 and 15145 in 2017). Overall, STI positivity has decreased in the youth age group (15-24 years), from $11.9 \%$ in 2012 to $3 \%$ in 2017 , and in antenatal care testing from $9.5 \%$ in 2012 to $1.1 \%$ in 2017. Chlamydia was the most common infection $(27.9 \%$ in 2012 and $22.9 \%$ in 2017), with fewer tests in 2017 compared to 2012 (2207 to 4951, respectively). Gonorrhoea is the second most common infection $(6.3 \%$ in 2012 and $10.0 \%$ in 2017), with more tests in recent years (2201 in 2017 compared to 208 in 2012). Hepatitis cases are low, with $2.6 \%$ positive rate in 2012 and $2.4 \%$ in 2017 for hepatitis B, and $1.1 \%$ in 2012 and $0.1 \%$ positive for hepatitis $C$. Testing for hepatitis $B$ and $C$ was higher in 2017 (9780 tests in 2012 and 22312 in 2017). In 2014 , there were 18 cases of syphilis from 3865 tests, and in 2017 there were 90 cases from 11418 tests.

\section{Results}

Positivity for STIs varied based on age and sex (Table 1 ). Chlamydia and gonorrhoea positivity rates were higher in the age group 15-19 years. In 2012, relative to those aged 15-19 years, those aged 20-24, 25-29 and $\geq 30$ years had significantly lower odds of testing positive for chlamydia (unadjusted odds ratio of $0.80,0.58$ and 0.32 , respectively). In 2017, relative to those aged 15-19 years, those aged $25-29$ years and $\geq 30$ years had 
Table 1. Odds of chlamydia, gonorrhoea, and hepatitis B and C infections by age and sex, 2012 and 2017

\begin{tabular}{|c|c|c|c|c|c|c|c|c|}
\hline \multirow[b]{2}{*}{2012} & \multicolumn{2}{|l|}{ Chlamydia } & \multicolumn{2}{|c|}{ Gonorrhoea } & \multicolumn{2}{|c|}{ Hepatitis B } & \multicolumn{2}{|c|}{ Hepatitis C } \\
\hline & OR $(95 \% \mathrm{Cl})^{\mathrm{C}}$ & $p$-value & OR $(95 \% \mathrm{Cl})^{\mathrm{C}}$ & $p$-value & OR $(95 \% \mathrm{CI})^{\mathrm{C}}$ & $p$-value & OR $(95 \% \mathrm{CI})^{\mathrm{C}}$ & $p$-value \\
\hline \multicolumn{9}{|l|}{ Sex ${ }^{a}$} \\
\hline Male & $1.05(0.742-1.48)$ & 0.79 & $15.73(3.37-73.42)$ & $0.00 *$ & $2.37(1.83-3.07)$ & $0.00 *$ & $9.31(1.27-68.44)$ & $0.01^{*}$ \\
\hline Female & 1 & & 1 & & 1 & & 1 & \\
\hline \multicolumn{9}{|l|}{ Age group ${ }^{b}$} \\
\hline $15-19$ & 1 & & 1 & & 1 & & 1 & \\
\hline $20-24$ & $0.80(0.67-0.96)$ & $0.01 *$ & $1.84(0.19-17.71)$ & 0.58 & $1.75(0.77-3.97)$ & 0.18 & $0.44(0.07-2.67)$ & 0.36 \\
\hline $25-29$ & $0.58(0.47-0.70)$ & $0.00 *$ & 0.97 (0.08-11.48) & 0.98 & $3.86(1.74-8.56)$ & $0.00 *$ & $0.57(0.09-3.43)$ & 0.53 \\
\hline$\geq 30$ & 0.32 & $0.00 *$ & $1.21(0.10-14.43)$ & 0.88 & $5.54(2.57-11.92)$ & $0.00 *$ & $1.28(0.29-5.58)$ & 0.74 \\
\hline 2017 & OR $(95 \% \mathrm{CI})^{\mathrm{C}}$ & $p$-value & OR $(95 \% \mathrm{Cl})^{\mathrm{C}}$ & $p$-value & OR $(95 \% \mathrm{Cl})^{\mathrm{C}}$ & $p$-value & OR $(95 \% \mathrm{CI})^{\mathrm{C}}$ & $p$-value \\
\hline \multicolumn{9}{|l|}{ Sex } \\
\hline Male & $0.91(0.65-1.26)$ & 0.55 & 7.10 (5.13-9.82) & $0.00 *$ & $2.48(1.95-3.17)$ & $0.00^{*}$ & $0.67(0.21-2.12)$ & 0.50 \\
\hline Female & 1 & & 1 & & 1 & & 1 & \\
\hline \multicolumn{9}{|l|}{ Age Group } \\
\hline $15-19$ & 1 & & 1 & & 1 & & - & - \\
\hline $20-24$ & $0.78(0.52-1.15)$ & 0.21 & $0.27(0.13-0.56)$ & $0.00 *$ & $2.56(0.77-8.49)$ & 0.11 & - & - \\
\hline $25-29$ & $0.55(0.36-0.82)$ & $0.00 *$ & $0.55(0.29-1.04)$ & 0.06 & $4.35(1.34-14.12)$ & $0.01^{*}$ & - & - \\
\hline$\geq 30$ & $0.27(0.17-0.41)$ & $0.00 *$ & $0.43(0.23-0.82)$ & $0.01 *$ & 11.82 (3.77-37.05) & $0.00 *$ & - & - \\
\hline \multicolumn{9}{|c|}{$\begin{array}{l}\text { a reporting only cases where sex is known } \\
\text { b reporting only cases where age is known } \\
\text { c extended Mantel-Haenszel } \chi^{2} \text { for trend, two-tailed sig. } \\
\text { * significant at } 0.05 \text { level } \\
\text { - insufficient cases for analysis } \\
\mathrm{Cl}=\text { confidence interval } \\
\mathrm{OR}=\text { odds ratio }\end{array}$} \\
\hline
\end{tabular}

significantly lower odds of testing positive (unadjusted odds ratio of 0.55 and 0.27 , respectively). No significant results were observed for sex in 2012 and 2017.

For gonorrhoea, compared with females, males had greater odds of testing positive in both 2012 and 2017 (unadjusted odds ratio of 15.7 and 7.1 , respectively). In 2012, positive tests did not differ significantly by age. In 2017, relative to those aged 15-19 years, those aged 20-24 years and those aged $\geq 30$ years had significantly lower odds of testing positive for gonorrhoea (unadjusted odds ratio of 0.27 and 0.55 , respectively).

For hepatitis B, compared with females, males had greater odds of testing positive in both 2012 and 2017 (unadjusted odds ratio of 2.4 and 2.5 , respectively). In both 2012 and 2017, relative to those aged 15-19 years, those aged $25-29$ and $\geq 30$ years had significantly greater odds of testing positive (2012: unadjusted odds ratio of 3.9 and 5.5, respectively; 2017: unadjusted odds ratio of 4.3 and 11.8 , respectively).

For hepatitis C, compared with females, males had significantly greater odds of testing positive in 2012, but not in 2017 (unadjusted odds ratio in 2012 of 9.3 and in 2017 of 0.67). In 2012, with respect to age, there was no difference in test positivity.

For syphilis, compared with females, males had lower odds of testing positive in both 2014 and 2017 (unadjusted odds ratio of 0.28 and 0.63 , respectively) (Table 2). In 2014, relative to those aged under 45 years, those $\geq 45$ years were 50 -fold more likely to test positive. In 2017, compared with those under 45 years, those aged $\geq 45$ years were 14 -fold more likely to test positive. For 2017, there were sufficient data to compare smaller 
Table 2. Odds of syphilis infection by age and sex, 2014 and 2017

\begin{tabular}{|c|c|c|c|c|}
\hline & Positive & Negative & OR $(95 \% \mathrm{Cl})^{\mathrm{C}}$ & $p$-value \\
\hline \multicolumn{5}{|l|}{2014} \\
\hline \multicolumn{5}{|l|}{$\operatorname{Sex}^{\mathrm{a}}$} \\
\hline Male & 6 & 476 & $0.28(0.11-076)$ & $0.01^{*}$ \\
\hline Female & 12 & 3371 & 1 & \\
\hline \multicolumn{5}{|c|}{ Age (years) ${ }^{b}$} \\
\hline $15-24$ & 0 & 1582 & - & - \\
\hline $25-34$ & 4 & 1492 & - & - \\
\hline $35-44$ & 1 & 486 & - & - \\
\hline$\geq 45$ & 9 & 128 & - & - \\
\hline$<45^{d}$ & 5 & 3560 & 1 & - \\
\hline$\geq 45$ & 9 & 128 & $50.06(16.54-151.51)$ & $0.00 *$ \\
\hline \multicolumn{5}{|l|}{2017} \\
\hline \multicolumn{5}{|l|}{ Sex $^{a}$} \\
\hline Male & 30 & 4934 & $0.63(0.40-0.97)$ & $0.04^{*}$ \\
\hline Female & 60 & 6190 & 1 & \\
\hline \multicolumn{5}{|c|}{ Age (years) ${ }^{\mathrm{b}}$} \\
\hline $15-24$ & 7 & 3437 & 1 & - \\
\hline $25-34$ & 16 & 4081 & $1.93(0.79-4.68)$ & 0.14 \\
\hline $35-44$ & 12 & 1892 & $3.11(1.22-7.92)$ & $0.01^{*}$ \\
\hline$\geq 45$ & 55 & 1055 & $25.60(11.62-56.38)$ & $0.00 *$ \\
\hline$<45^{d}$ & 35 & 9410 & 1 & \\
\hline$\geq 45$ & 55 & 1055 & $14.02(9.13-21.52)$ & $0.00 *$ \\
\hline \multicolumn{5}{|c|}{$\begin{array}{l}\text { a reporting only cases where sex is known } \\
\text { b reporting only cases where age is known } \\
\text { ' extended Mantel-Haenszel } \chi^{2} \text { for trend, two-tailed sig. } \\
\text { d insufficient data for age groups } 15-44 \text {, all }<45 \text { were compared to } 45+\text { in } 2014 \\
\text { * significant at } 0.05 \text { level. } \\
\mathrm{Cl}=\text { confidence interval } \\
\mathrm{OR}=\text { odds ratio }\end{array}$} \\
\hline
\end{tabular}

age groups. Compared to those aged 15-24 years, all older age groups had greater odds of a positive syphilis test (unadjusted odds ratio of 3.1 for those aged 35-44 years and 25.6 for those aged $\geq 45$ years).

\section{DISCUSSION}

This analysis finds similar positivity rates to seroprevalence estimations, and it provides gender and age trends in positivity. As expected, young people generally had greater odds of testing positive for chlamydia (high prevalence in both 2012 and 2017) and gonorrhoea, while older age groups were significantly more likely to be positive for hepatitis $\mathrm{B}$ and $\mathrm{C}$. This is a common trend as older age groups have longer windows of exposure to viral infections and have had more routine screening over their lifetime, while bacterial infections are more episodic and frequent with young people. Older individuals may also have lower vaccine coverage than younger ones for hepatitis $B$ virus and hepatitis $C$ virus. However, older age groups had greater odds of testing positive for syphilis.

The high positivity rates for chlamydia in 2012 and 2017, in particular, are of great concern. This is due to: 1) the lack of population interventions to reduce chlamydia prevalence; 2) diminishing prioritization of STIs; and 3) the social stigma surrounding sex and STIs that deters individuals from seeking health services and makes delivering comprehensive sexual health education to young people difficult. In a small and close-knit population, seeking services anonymously for taboo conditions is almost impossible and can result in social consequences. ${ }^{12}$

It is also unusual for syphilis to be more common in older age groups. We suggest this anomaly may be due to untreated syphilis cases being detected in late tertiarystage years after an initial infection, when advanced symptoms resurface. Reports indicate a significant increase in infections between 2015 and 2016. ${ }^{11}$ The odds ratio of syphilis infections was higher in those age $\geq 45$ years both in 2014 and 2017. In addition to older generations being diagnosed in late stages, these results could illuminate a health system-level gap in case detection for syphilis.

From a cultural perspective, older individuals in Samoa are also afforded more social authority, allowing them more agency to freely pursue sexual activity and attend health services. Sexual activity in younger individuals, especially those not married, is considered a more serious taboo and is socially discouraged. ${ }^{12}$ Additionally, older generations had comparatively less access to sexual health education and prevention. All of these factors would increase the odds of infection in older populations.

In Samoa, government-level STI prevention has focused on young people aged 15-24 years as a high-risk group with the largest burden of infection. ${ }^{11}$ This study confirms such trends for chlamydia and gonorrhoea. However, syphilis interventions may require age-specific strategies for older populations. These results highlight the need to design age-specific interventions for case detection and prevention in older populations. 


\section{Limitations}

The HIV and STI Surveillance Database at the Ministry of Health captures results of all diagnostic tests nationally, with the exception of rapid screening kits. Therefore, the data represent all patients (public, private, routine and suspect) in the country accessing clinical services who received a diagnostic test, excluding screened cases not referred for diagnostics and symptomatic cases treated presumptively. There is selection bias from suspect cases referred to testing by clinicians. Additional studies are needed to estimate the true population prevalence of populations that do not attend services. Information on the stage of infection, exposure and result per assay is not captured.

Each case in the database represents an episode of STI infection, with the possibility of a single patient having multiple episodes. However, anecdotal observations from the Ministry of Health indicate that patients often delay seeking testing and care until infections enter an advanced stage. For instance, only $52 \%$ of children under age 5 years with a fever were brought to a health facility for care or advice. ${ }^{13}$ This late attendance suggests it may be unlikely for patients to report to services more than once a year for STIs.

To obtain a sufficient sample size for syphilis, data were taken from 2014, and age groups were recategorized into 10-year age groups before running the calculations by age and sex, which resulted in larger confidence intervals as the age group categories were less distinct from each other. Additionally, the positivity for TPHA/RPR may indicate chronic yaws infections in older adults, as opposed to syphilis; however, yaws was declared eliminated in the 1960 s. ${ }^{14}$

\section{CONCLUSION}

The unexpectedly higher odds of syphilis positivity among adults aged $\geq 45$ years compared to $15-24$ years, as well as the persistently high rates of chlamydia infections between 2012 and 2017, highlight a need to re-strategize the promotion and delivery of STI testing and care services in Samoa. Prevention services and strategies should be age-specific, with further assessment of the different barriers for older and younger patients.

\section{Acknowledgments}

We would like to thank all national laboratory staff at the National Reference Laboratory, Tupua Tamasese Meaole (TTM) Hospital in Upolu, and Laboratory Services, MT2, Savai'i for their testing and data collection over the years that made this analysis possible, especially Lupe Isaia, Laboratory Scientist of Laboratory Services of the TTM Hospital in Apia, Samoa.

In addition, we would like to express our appreciation to all rural district health centres and hospitals in Upolu and Savai'i, whose efforts provided valuable assistance in case reporting.

We also would like to thank Barbara Telfer, visiting epidemiologist, for her feedback in the preparation of this manuscript.

Finally, we would like to thank Serafi Moa, Senior Nurse Specialist, and Dr Sarah Brown, Specialist Physician, of the Communicable Disease Clinic (TTM Hospital, Apia) for the clinical work and dedication to reaching people with services they have provided over the years.

\section{References}

1. 2016 Census brief no. 1: Population snapshot and household highlights. Apia: Samoa Bureau of Statistics; 2017.

2. Sullivan EA, Koro S, Tabrizi S, Kaldor J, Poumerol G, Chen S, et al. Prevalence of sexually transmitted diseases and human immunodeficiency virus among women attending prenatal services in Apia, Samoa. Int J STD AIDS. 2004 Feb;15(2):116-9. doi:10.1258/095646204322764316

3. Cliffe SJ, Tabrizi S, Sullivan EA; Pacific Islands Second Generation HIV Surveillance Group. Chlamydia in the Pacific region, the silent epidemic. Sex Transm Dis. 2008 Sep;35(9):801-6. doi:10.1097/ OLQ.0b013e318175d885

4. Walsh MS, Hope E, Isaia L, Righarts A, Niupulusu T, Temese SV, et al. Prevalence of Chlamydia trachomatis infection in Samoan women aged 18 to 29 and assessment of possible risk factors: a community-based study. Trans R Soc Trop Med Hyg. 2015 Apr;109(4):245-51. doi:10.1093/trstmh/trv014

5. Menon S, Stansfield SH, Walsh M, Hope E, Isaia L, Righarts AA, et al. Sero-epidemiological assessment of Chlamydia trachomatis infection and sub-fertility in Samoan women. BMC Infect Dis. 2016 Apr 21;16(1):175. doi:10.1186/s12879-016-1508-0

6. HIV/STI/TB National Programme, Samoa. 2018 HIV \& STI surveillance report January to December (internal report). Apia: Ministry of Health, Samoa; 2018.

7. Gomez GB, Kamb ML, Newman LM, Mark J, Broutet N, Hawkes SJ. Untreated maternal syphilis and adverse outcomes of pregnancy: a systematic review and meta-analysis. Bull World Health Organ. 2013 Mar 1;91(3):217-26. doi:10.2471/BLT.12.107623 
8. Garcia Garcia L, Gonzalez-Escalada A, Ariza Megia MC, Gil-de-Migue A. Syphilis: An epidemiological review. Curr Women's Health Rev. 2012;8(3):231-41. doi:10.2174/157340412803760694

9. Viera AJ. Odds ratios and risk ratios: what's the difference and why does it matter? South Med J. 2008 Jul;101(7):730-4. doi:10.1097/ SMJ.0b013e31817a7ee4

10. Schmidt CO, Kohlmann T. When to use the odds ratio or the relative risk? Int J Public Health. 2008;53(3):165-7. doi:10.1007/s00038008-7068-3

11. Ministry of Health. Samoa Global AIDS Monitoring Report 2018. Apia, Samoa. UNAIDS. 1 July, 2018. Available from: https://www. unaids.org/sites/default/files/country/documents/WSM_2018 countryreport.pdf
12. Howells ME, Lynn CD, Weaver LJ, Langford-Sesepesara M, Tufa J. Zika virus in American Samoa: challenges to prevention in the context of health disparities and non-communicable disease. Ann Hum Biol. 2018 May;45(3):229-38. doi:10.1080/03014460.201 8.1465594

13. Samoa Bureau of Statistics. Demographic Health Survey 2014. Government of Samoa; 2015.

14. Capuano C, Ozaki M. Yaws in the Western pacific region: a review of the literature. J Trop Med. 2011:2011:642832. doi:10.1155/2011/642832 